\title{
FRAMES ASSOCIATED WITH AN ABELIAN $l$-GROUP
}

\author{
JAMES J. MADDEN
}

\begin{abstract}
Every archimedean $l$-group (lattice-ordered group) with weak unit is shown to be isomorphic to a sub-l-group of the $l$-group of continuous realvalued functions on a Tychonoff locale canonically associated with the $l$-group. This strengthens the classical Yosida representation theorem in a useful way. The proof uses methods from universal algebra and is constructive.
\end{abstract}

\section{INTRODUCTION}

The Yosida Representation Theorem states that every archimedean l-group with weak unit is isomorphic to a sub-l-group of the partial $l$-group of all continuous $\mathbb{R} \cup\{ \pm \infty\}$-valued functions with dense domains of reality on a compact Hausdorff space. J. Vermeer and this author showed in a previous paper $\left[\mathrm{MV}_{2}\right]$ that this implies the following "localic Yosida theorem:" every archimedean $l$-group with weak unit is isomorphic to a sub- $l$-group of the $l$-group of all continuous real-valued functions on a regular Lindelöf locale (the "Yosida locale"). The paper in hand first presents a proof of the localic Yosida theorem which does not depend on the classical theorem and then applies ideas involved in the proof to study certain functors from $l$-groups to frames and to $\sigma$-frames which are related to the Yosida representation. The perspective taken here is from universal algebra, in contrast to the topological point of view which was adopted in $\left[\mathrm{MV}_{2}\right]$. This proves to be much more transparent and informative.

One advantage of the direct proof of the localic Yosida theorem is aesthetic. The Yosida locale was constructed in $\left[\mathrm{MV}_{2}\right]$ by removing points-in some cases all of them-from the classical Yosida space. Effort is obviously wasted in manufacturing points which will later only have to be discarded. There are several practical advantages in a direct proof also, especially in terms of clarity and concreteness. For example, a certain functor from l-groups to $\sigma$ frames which plays a crucial role in $\left[\mathrm{MV}_{2}\right]$ is described there as a composite of functors which themselves require involved definitions (see after 3.1 in $\left[\mathrm{MV}_{2}\right]$ ). In contrast, in this paper, we give a transparent description of this functor by means of generators and relations $(\$ 7)$ by exploiting some of the machinery developed in the direct proof of the localic Yosida theorem. Another practical gain is the light which the direct proof sheds upon the relationship between the

Received by the editors August 25, 1988 and, in revised form, February 12, 1990.

1980 Mathematics Subject Classification (1985 Revision). Primary 46A40, 06F20, 54H10; Secondary $18 \mathrm{C} 05,46 \mathrm{R} 05,06 \mathrm{~B} 35$.

Key words and phrases. Yosida representation, archimedean $l$-group, relatively uniform convergence, frame, locale, $\sigma$-frame. 
topologies of the Yosida space and of the Yosida locale and various lattices of $X$-complete $l$-ideals, where " $X$ " refers to some prescribed class of suprema in the l-group. In particular, we get an easy Corollary (6.3) saying that the weakunital archimedean $l$-groups closed under countable composition are classified up to isomorphism by their lattices of (relatively) uniformly complete $l$-ideals. A third practical gain is the indication of ways in which localic methods might be extended to the category of all archimedean l-groups. The approach of $\left[\mathrm{MV}_{2}\right]$ depends on functorial features of the classical Yosida theorem which do not work when weak units are absent or not preserved by the morphisms. In the present paper, we find functors $(\mathscr{Y}$ of $(3.1)$ and $\sigma-\mathscr{Y}$ of $\S 7)$ which generalize the functors of $\left[\mathrm{MV}_{2}\right]$ in what appear to be useful ways.

In writing a direct proof of the localic Yosida theorem, something very pleasant (and not wholly unanticipated) was noted, namely that the axiom of choice was never used and in fact the proof was valid constructively. In this paper, pains have been taken to present constructive arguments for all results contained in $\S \S 1$ through 4 . This includes a proof that every archimedean l-group with weak unit admits an injection into the $l$-group of all real-valued functions on a completely regular locale. The constructive existence of the localic representation means that it is actually a much stronger tool than the classical representation, since it exists in any topos with a natural number object. As is well known, this degree of generality is quite relevant to "down to earth" mathematics in the usual set-theoretic universe. For example, we automatically have a new representation theorem for sheaves of $l$-groups. This is completely different from Keimel's representation of $l$-groups by sections of sheaves and means (roughly) that a continuously varying representation can be chosen for a continuously varying family of $l$-groups. However, we leave the exploration of such applications for another occasion.

The algebraic methods developed in this paper to replace the topological methods of $\left[\mathrm{MV}_{2}\right]$ provide ways of simplifying parts of the complicated theory of $l$-group extensions which has been worked out by Ball and Hager and Ball-Hager-Neville in a long series of papers. Some results along these lines will appear in $\left[\mathrm{M}_{2}\right]$. The efficiency of the frame-theoretic approach suggests the possibility of a much broader role for frames in connection with parts of analysis and measure theory, especially where representations of abstract algebras as algebras of continuous functions come into play. As opposed to other work applying frames to analysis, e.g. Mulvey [Mu], the point to be made here is not primarily that theorems valid in a topos can be proved, but just that frame methods are extraordinarily efficient and clear. This echoes a position stated convincingly by J. R. Isbell at the 1988 Northeast Conference on General Topology. To the objection which may occur to some readers-namely, that the classical Yosida theorem can be proved in two pages, while the localic version presented seems to require a much longer proof-the reply is this: the starting point in our proof is the definition of an $l$-group and a few elementary properties of frames. If $\S 2$ is viewed as part of the proof, then our starting point does not even include any of the elementary properties of the real numbers. Familiar proofs of the classical version depend on extensive background in archimedean $l$-groups, the theory of $l$-groups ideals and lots of elementary, but lengthy to prove, facts about the real number system. 
Added in proof. A discussion of the main motivating ideas of the present work has appeared in $\left[\mathrm{M}_{1}\right]$.

The contents of this paper are as follows. Section 1 reviews the presentation of frames by generators and relations. Rather than using the terminology of "sites" (as in [J]) or the approach via propositional theories (as in [BM]), a language emphasizing the analogy with universal algebra has been adopted. This seemed most likely to be immediately accessable to the intended audience. The three examples of $\S 1$ are well known. In $\S 2$, a constructive proof that the locale of real numbers is an $l$-group is sketched. Such proofs are well known to many category-theorists, but it is difficult to extract a proof from published sources. Section 3 introduces the system of relations needed to define the Yosida locale of an arbitrary l-group. In $\S 4$, the Yosida locale and the localic representation of a pointed $l$-group $(A, e)$ are defined. It is shown constructively that the representation is an $l$-homomorphism, that the Yosida locale is completely regular and that the representation has zero kernel when $A$ is archimedean and $e$ is a weak unit. In $\S 5$, we relate the Yosida locale to the frame of (relatively) uniformly closed $l$-ideals. Section 6 gives generators and relations for the topology of the classical Yosida space, then uses this to show that the Yosida locale, as defined in $\S 4$, is the same object treated in $\left[\mathrm{MV}_{2}\right]$. Section 7 discusses the $\sigma$-frames determined by the same generators and relations used to define the Yosida space and the Yosida locale.

The reader unfamiliar with frames and locales can very rapidly pick up the background knowledge we assume by reading selected parts of P. T. Johnstone's book [J], especially the first sections of Chapter II. In order to fix notation, we repeat a bit. A frame is a lattice with top $T$ and bottom $\perp$ in which every subset has a supremum and in which the following distributive law holds:

$$
a \wedge \bigvee B=\bigvee\{a \wedge b \mid b \in B\}
$$

A morphism of frames is a map between frames which preserves top, bottom, finite infima and all suprema. The category of frames is denoted Frm. Its formal opposite, the category of locales, is denoted Loc. If $F$ is a frame, the corresponding locale is denoted $L(F)$. If $L$ is a locale, the corresponding frame is denoted $O(L)$. If $X$ is a topological space, its frame of open subsets is also denoted $O(X)$. Note that in [J], Johnstone would write just $F$ 'where we write $L(F), L$ where we write $O(L)$ and $\Omega(X)$ where we write $L O(X)$. The symbol $\&$ denotes the initial object in the category of frames, i.e. the two element frame $\{T, \perp\}$.

All l-groups in this paper are abelian and we use additive notation. An abelian $l$-group is an abelian group equipped with a semilattice operation $\mathrm{V}$ for which $x+(y \vee z)=(x+y) \vee(x+z)$ for all elements $x, y$, and $z$. (This is equivalent to the definition, in [BKW], and indeed constructively so.) In an abelian $l$-group, the operation $\wedge$ is defined by $x \wedge y=-(-x \vee-y)$.

\section{FRAMES BY GENERATORS AND RELATIONS}

In [J, II.2.11], Johnstone describes and justifies a method of specifying frames by giving generators and relations. We will use this repeatedly below, so we describe the procedure in this section. We have chosen here to use terminology which emphasizes the analogy with familiar procedures from universal algebra. 
What we say may easily be translated into the terminology of Johnstone's book, if one desires.

Let $F(E)$ be the free frame on the set $E$. If $f: E \rightarrow G$ is a set map from $E$ to a frame $G$, then $\bar{f}$ denotes the unique induced frame morphism from $F(E)$ to $G$. Suppose $R$ is any subset of $F(E) \times F(E)$. One may view $R$ as a set of equations between frame words in the symbols $E$. The frame freely generated by $E$ subject to the relations $R$ is a set map $j$ from $E$ to a frame (for the time being unnamed) such that:

(1) If $(x, y) \in R$, then $\bar{j}(x)=\bar{j}(y)$.

(2) If $\varphi_{0}$ is a set map from $E$ to a frame and $\bar{\varphi}_{0}(x)=\bar{\varphi}_{0}(y)$ for all $(x, y) \in$ $R$, then there is a unique frame morphism $\varphi$ such that $\varphi \circ j=\varphi_{0}$.

It is easy to see that for the codomain of $j$ we may take $F(E) / K(R)$, where $K(R)$ is the frame congruence generated by $R$, and it is permissible to refer to this frame as the frame freely generated by $E$ subject to $R$. Generally, we choose a suggestive symbol other than $F(E) / K(R)$ to denote the codomain of $j$. It is convenient when specifying a frame by generators and relations to give $R$ by stating equations satisfied in the codomain of $j$ by the images of the elements of $E$, as in the following examples.

1.1. Example. We describe coproducts in Frm. Let $A$ and $B$ be frames and let $A \times B$ be the product of the (underlying sets) of $A$ and $B$. Let $j: A \times B \rightarrow$ $A \otimes B$ be the frame freely generated by $A \times B$ subject to the following relations:

$\left(\mathrm{C}_{1}\right) j((\mathrm{~T}, \mathrm{~T}))=\mathrm{T} ; j((a, \perp))=j((\perp, b))=\perp$,

$\left(\mathrm{C}_{2}\right) \quad j((a, b)) \wedge j\left(\left(a^{\prime}, b^{\prime}\right)\right)=j\left(\left(a \wedge a^{\prime}, b \wedge b^{\prime}\right)\right)$,

$\left(\mathrm{C}_{3}\right) \bigvee\{j((x, b)) \mid x \in X\}=j((\bigvee X, b))$;

$\bigvee\{j((a, y)) \mid y \in Y\}=j((a, \bigvee Y))$.

This is analogous to the definition of the tensor product of rings. The generator $j((a, b))$ will often be written $a \otimes b$, and every element of $A \otimes B$ is of the form $\bigvee\left\{a_{\lambda} \otimes b_{\lambda} \mid \lambda \in I\right\}$. Together with the morphisms $a \mapsto a \otimes T(a \in A)$ and $b \mapsto \mathrm{T} \otimes b \quad(b \in B), A \otimes B$ is the coproduct of $A$ and $B$ in Frm. More details can be found in $[\mathrm{J}]$. For a deep exploration of the analogy between frames and rings, see [JT].

1.2. Example. We shall give a presentation of a certain frame $\mathscr{R}$ which will be the frame-theoretic counterpart of the topological l-group of real numbers. Let $\mathbb{Q}$ be the totally ordered set of rational numbers. Elements of $\mathbb{Q} \times \mathbb{Q}$ will be written with sharp brackets, e.g., $\langle 0,1\rangle$. Define $j: \mathbb{Q} \times \mathbb{Q} \rightarrow \mathscr{R}$ to be the frame freely generated by $\mathbb{Q} \times \mathbb{Q}$ subject to the following relations:
$\left(\mathbf{R}_{1}\right)$ If $b \leq a, j(\langle a, b\rangle)=\perp$,
$\left(\mathbf{R}_{2}\right) j(\langle a, b\rangle) \wedge j(\langle c, d\rangle)=j(\langle\max \{a, c\}, \min \{b, d\}\rangle)$,
$\left(\mathrm{R}_{3}\right)$ if $a \leq c<b \leq d, j(\langle a, b\rangle) \vee j(\langle c, d\rangle)=j(\langle a, d\rangle)$,
$\left(\mathbf{R}_{\mathbf{4}}\right) \bigvee\{j(\langle x, y\rangle) \mid \bar{a}<x<y<b\}=j(\langle a, b\rangle)$,
$\left(\mathrm{R}_{5}\right) \bigvee\{j(\langle x, y\rangle) \mid\langle x, y\rangle \in \mathbb{Q} \times \mathbb{Q}\}=\mathrm{T}$.

For convenience, the generator $j(\langle a, b\rangle)$ will be written just $(a, b)$. Also, we will write $(-\infty, b)$ and $(a, \infty)$ as abbreviations for $\bigvee\{(x, b) \mid x \in \mathbb{Q}\}$ and $\bigvee\{(a, y) \mid y \in \mathbb{Q}\}$ respectively.

If $\mathbb{R}$ is the space of real numbers and $\varphi_{0}: \mathbb{Q} \times \mathbb{Q} \rightarrow O(\mathbb{R})$ is defined by letting $\varphi_{0}(\langle a, b\rangle)=\{x \in \mathbb{R} \mid a<x<b\}$, then $\varphi_{0}$ satisfies conditions $\left(\mathbf{R}_{1}\right)-\left(\mathbf{R}_{5}\right)$ and hence extends to a frame morphism $\varphi: \mathscr{R} \rightarrow O(\mathbb{R})$. The Heine-Borel theorem 
can be used to show that $\varphi$ is an isomorphism, see [FH]. Interestingly, however, it is not constructively valid that $\varphi$ is an isomorphism, see [FH or J]. In stating and proving the constructive version of the Yosida theorem, it turns out to be $\mathscr{R}$ and not $O(\mathbb{R})$ which has the appropriate properties. The frame $O(\mathbb{R})$ is not very useful to us.

1.3. Example. The frame of all $l$-ideals of an abelian $l$-group can be presented by generators and relations. Let $A$ be an abelian $l$-group and let $A^{+}=\{a \in$ $A \mid a \geq 0\}$. Let $\mathscr{I}(A)$ be the frame freely generated by $A^{+}$subject to the following relations:

(I $\left.\mathrm{I}_{1}\right) j(0)=\perp$,

(I $\left.\mathrm{I}_{2}\right) j(a \wedge b)=j(a) \wedge j(b)$

(I $\left.\mathrm{I}_{3}\right) j(a+b)=j(a) \vee j(b)$

(I4) $j(a \vee b)=j(a) \vee j(b)$.

Let $l-\operatorname{Idl}(A)$ denote the set of all $l$-ideals of $A$. By [BKW], $l-\operatorname{Idl}(A)$ is a frame in the order induced by (set-theoretic) inclusion. For each $a \in A^{+}$, let $i_{0}(a)$ be the smallest $l$-ideal of $A$ containing $a$. By arguments similar to those of [J, pp. 211-212] (see also [BKW]) the map $i_{0}$ satisfies relations $\left(\mathrm{I}_{1}\right)-$ $\left(\mathrm{I}_{4}\right)$, so it determines a frame morphism $i: \mathscr{I}(A) \rightarrow l$-Idl $(A)$. Obviously, $i(\bigvee\{j(x) \mid x \in X\})$ is the $l$-ideal generated by $X$, so $i$ is surjective. In order to describe the kernel of $i$, first observe that if $y \in A^{+}$belongs to the $l$ ideal generated by $X \subseteq A^{+}$, then there is some finite sequence $x_{1}, \ldots, x_{n}$ of elements of $X$ such that $y \leq x_{1}+\cdots+x_{n}$, and thus $j(y) \leq j\left(x_{1}\right) \vee \cdots \vee j\left(x_{n}\right)$. It follows that if subsets $X$ and $Y$ generate the same $l$-ideal of $A$, then

$$
\bigvee\{j(x) \mid x \in X\}=\bigvee\{j(y) \mid y \in Y\} .
$$

Thus, the only elements of $\mathscr{I}(A)$ which are identified by the morphism $i$ are the top element of $\mathscr{I}(A)$ and $\bigvee\left\{j(a) \mid a \in A^{+}\right\}$. Observe then that the interval $\left[\perp, \bigvee\left\{j(a) \mid a \in A^{+}\right\}\right] \subseteq \mathscr{I}(A)$ is order isomorphic to $l$ - $\operatorname{Idl}(A)$.

We have not included the relation $\mathrm{T}=\bigvee\left\{j(a) \mid a \in A^{+}\right\}$among the defining identities for $\mathscr{I}(A)$ for a good reason. Under the definition we have given, $\mathscr{I}$ is a functor. If $\varphi: A \rightarrow B$ is a morphism of abelian $l$-groups, then $j_{B} \circ \varphi$ satisfies relations $\left(\mathrm{I}_{1}\right)-\left(\mathrm{I}_{4}\right)$ so there is a frame morphism $\mathscr{I}(\varphi): \mathscr{I}(A) \rightarrow \mathscr{I}(B)$ such that $\mathscr{I}(\varphi) \circ j_{A}=j_{B} \circ \varphi$. The additional relation mentioned above, however, is not compatible with all $l$-homomorphisms. Consider the zero morphism from $\mathbb{Z}$ to $\mathbb{Z}$. The $l$-homomorphisms with which the relation is compatible are precisely the majorizing $l$-homomorphisms. See [HM] for much more about these special maps.

\section{2. $L(\mathscr{R})$ IS AN $l$-GROUP OBJECT IN LOC}

We switch momentarily to locale language for convenience. We shall show that $\operatorname{Hom}_{\mathrm{Loc}}(X, L(\mathscr{R}))$ is an $l$-group in a natural way. This is equivalent to the existence of certain Loc morphisms defined on products of $L(\mathscr{R})$ with itself which have properties analogous to those satisfied by $l$-group operations. These will be called "l-group structure morphisms" for $L(\mathscr{R})$. The idea is made clearer below. The results of this section are basically well known (for example, $[\mathrm{BM}]$ refers to the lattice-ordered ring structure of $\mathscr{R}$ ), and no claim of orginality is made. It is difficult to track down in other published sources the 
details which we discuss here, however, and since these details are needed in subsequent sections the inclusion of this expositional material seems necessary.

If we were to accept the nonconstructive assertion $\mathscr{R}=O(\mathbb{R})$, the existence of $l$-group structure morphisms for $L(\mathscr{R})$ could be established as follows. By the local compactness of $\mathbb{R}, L O(\mathbb{R} \times \mathbb{R}) \cong L O(\mathbb{R}) \times_{l} L O(\mathbb{R})$, see $[\mathrm{J}]$. Thus, addition $+: \mathbb{R} \times \mathbb{R} \rightarrow \mathbb{R}$ induces a locale morphism $a: L O(\mathbb{R}) \times_{l}$ $L O(\mathbb{R}) \rightarrow L O(\mathbb{R})$. The reader can easily check that by means of this morphism, $\operatorname{Hom}_{\mathrm{Loc}}(, L O(\mathbb{R}))$ is a functor from Loc to the category of groups. The sum of two morphisms $f, g \in \operatorname{Hom}_{\mathrm{Loc}}(X, L O(\mathbb{R}))$ is $a \circ(f, g)$. In a similar way, the supremum operation in $\mathbb{R}$ induces a locale morphism $s: L O(\mathbb{R}) \times_{l}$ $L O(\mathbb{R}) \rightarrow L O(\mathbb{R})$, and this can be used to produce a semilattice operation in $\operatorname{Hom}_{L o c}(X, L O(\mathbb{R}))$ which makes it an l-group.

Even nonconstructively the existence of a group structure morphism is not trivial, since the local compactness of $\mathbb{R}$ is crucial. In the case of the topological group $\mathbb{Q}$ of rational numbers, $L O(\mathbb{Q} \times \mathbb{Q})$ is a proper sublocale of $L O(\mathbb{Q}) \times_{l}$ $L O(\mathbb{Q})$, and addition does not induce a semigroup structure morphism from $L O(\mathbb{Q}) \times{ }_{l} L O(\mathbb{Q})$ to $L O(\mathbb{Q})$. This has been observed independently by several people, including F. Kirwan in her 1981 Cambridge thesis, who was possibly the first.

Constructively, it is not possible to use the algebraic operations on $\mathbb{R}$ to obtain structure morphisms for $\mathscr{R}$. Instead, we proceed as follows. Using the notation from Example 1.2, we define maps $\alpha_{0}: \mathbb{Q} \times \mathbb{Q} \rightarrow \mathscr{R} \otimes \mathscr{R}, \sigma_{0}: \mathbb{Q} \times \mathbb{Q} \rightarrow$ $\mathscr{R} \otimes \mathscr{R}, \eta_{0}: \mathbb{Q} \times \mathbb{Q} \rightarrow \mathscr{R}$ and $\varepsilon_{0}: \mathbb{Q} \times \mathbb{Q} \rightarrow \mathcal{L}$ by the rules:

$\alpha_{0}(\langle a, b\rangle)=\bigvee\{(s, t) \otimes(u, v) \mid a \leq s+u \& t+v \leq b\}$,

$\sigma_{0}(\langle a, b\rangle)=(-\infty, b) \otimes(a, b) \vee(a, b) \otimes(-\infty, b)$,

$\eta_{0}(\langle a, b\rangle)=(-b,-a)$,

$\varepsilon_{0}(\langle a, b\rangle)=$ the truth-value of the assertion: " $a<0 \& 0<b$."

We shall prove the following lemmas:

2.1. Lemma. Each of the maps $\alpha_{0}, \sigma_{0}, \eta_{0}$ and $\varepsilon_{0}$ satisfies conditions $\left(\mathbf{R}_{1}\right)$ $\left(\mathbf{R}_{5}\right)$, hence each extends to a frame morphism with domain $\mathscr{R}$.

The extensions will be denoted $\alpha, \sigma, \eta$ and $\varepsilon$ respectively. In order to state the next lemma, we introduce the following auxiliary morphisms:

$\tau=$ the unique morphism $\& \rightarrow \mathscr{R}$,

$\xi: \mathscr{R} \otimes \mathscr{R} \rightarrow \mathscr{R} \otimes \mathscr{R} ; U \otimes V \mapsto V \otimes U$,

$\nabla: \mathscr{R} \otimes \mathscr{R} \rightarrow \mathscr{R} ; U \otimes V \mapsto U \wedge V$ (codiagonal).

2.2. Proposition. The morphisms $\alpha, \sigma, \eta$ and $\varepsilon$ satisfy the following laws, making $\mathscr{R}$ into a commutative co-l-group in Frm with addition $\alpha$, join $\sigma$, minus $\eta$ and zero $\varepsilon$ :

(i) $(\alpha \otimes \mathrm{id}) \circ \alpha=(\mathrm{id} \otimes \alpha) \circ \alpha$ (associativity),

(i) $\xi \circ \alpha=\alpha$ (commutativity),

(iii) $(\varepsilon \otimes \mathrm{id}) \circ \alpha=\mathrm{id}$ (identity),

(iv) $\nabla \circ(\eta \otimes \mathrm{id}) \circ \alpha=\tau \circ \varepsilon$ (inverses),

(v) $(\sigma \otimes \mathrm{id}) \circ \sigma=(\mathrm{id} \otimes \sigma) \circ \sigma$ (associativity),

(vi) $\xi \circ \sigma=\sigma$ (commutativity),

(vii) $\nabla \circ \sigma=$ id (idempotence),

(viii) $(\mathrm{id} \otimes \sigma) \circ \alpha=(\nabla \otimes \mathrm{id} \otimes \mathrm{id}) \circ(\mathrm{id} \otimes \xi \otimes \mathrm{id}) \circ(\alpha \otimes \alpha) \circ \sigma$ (distributivity).

As the reader probably anticipates, the proofs of 2.1 and 2.2 are quite long 
but mostly routine. We shall include enough to show the typical form the verifications take, selecting only the more interesting parts of the proof.

Partial proof of 2.1. We show that $\alpha_{0}$ satisfies $\left(\mathbf{R}_{1}\right)-\left(\mathbf{R}_{5}\right)$. Conditions $\left(\mathbf{R}_{1}\right)$ and $\left(\mathbf{R}_{5}\right)$ are obvious. For $\left(\mathbf{R}_{2}\right)$, let

$$
\begin{aligned}
A & =\left\{\left(\max \left\{s_{1}, s_{2}\right\}, \min \left\{t_{1}, t_{2}\right\}\right) \otimes\left(\max \left\{u_{1}, u_{2}\right\}, \min \left\{v_{1}, v_{2}\right\}\right) \mid\right. \\
& \left.\subseteq \mathscr{R} \otimes \mathscr{R} \quad a \leq s_{1}+u_{1} \& t_{1}+v_{1} \leq b \& c \leq s_{2}+u_{2} \& t_{2}+v_{2} \leq d\right\}
\end{aligned}
$$

and let

$$
B=\{(s, t) \otimes(u, v) \mid \max \{a, c\} \leq s+u \& t+v \leq \min \{b, d\}\} .
$$

Then $\alpha_{0}(\langle a, b\rangle) \wedge \alpha_{0}(\langle c, d\rangle)=\bigvee A$ and $\alpha_{0}(\langle\max \{a, c\}, \min \{b, d\}\rangle)=\bigvee B$. The reader should have no difficulty verifying that $A$ and $B$ have the same elements.

$\left(\mathbf{R}_{3}\right)$ Claim. If $q>0$ is any rational and $(a, b)$ is a generator of $\mathscr{R}$, then $(a, b)=\bigvee\{(x, y) \mid a<x<y<b \& y-x \leq q\}$. This follows from $\left(\mathbf{R}_{3}\right)$ and $\left(\mathbf{R}_{4}\right)$ (for $j$ ) because for any rationals $a^{\prime}<b^{\prime}$ there exist finite sequences of rationals $a^{\prime}=x_{1}<x_{2}<\cdots<x_{n}$ and $y_{1}<y_{2}<\cdots<y_{n}=b^{\prime}$ with $x_{i+1}<y_{i}$ and $y_{i}-x_{i}<q$. To prove $\left(\mathrm{R}_{3}\right)$ for $\alpha_{0}$, let $a \leq c<b \leq d$ be any rationals and put $q=\frac{1}{2}(b-c)$. Consider the set $S=\{(s, t) \otimes(u, v) \mid a \leq s+u \& t+v \leq$ $d \& t-s<q \& v-u<q\}$. By the claim, $\bigvee S=\alpha_{0}(\langle a, d\rangle)$. Now take any $(s, t) \otimes(u, v) \in S$. If $s+u \geq c$, then $(s, t) \otimes(u, v) \leq \alpha_{0}(\langle c, d\rangle)$. On the other hand, if $s+u \leq c$, then $t+v \leq s+u+2 q \leq b$ so $(s, t) \otimes(u, v) \leq \alpha_{0}(\langle a, b\rangle)$. Thus $\alpha_{0}(\langle a, d\rangle)=\alpha_{0}(\langle a, b\rangle) \vee \alpha_{0}(\langle c, d\rangle)$.

$\left(\mathbf{R}_{4}\right) \quad \alpha_{0}(\langle a, b\rangle)=\bigvee\left\{\left(s^{\prime}, t^{\prime}\right) \otimes\left(u^{\prime}, v^{\prime}\right) \mid \exists s, t, u, v: s<s^{\prime}<t^{\prime}<t \& u<u^{\prime}<\right.$ $\left.v^{\prime}<v \& a \leq s+u \& t+v \leq b\right\}=\bigvee\left\{\left(s^{\prime}, t^{\prime}\right) \otimes\left(u^{\prime}, v^{\prime}\right) \mid \exists a^{\prime}, b^{\prime}: a<a^{\prime}<b^{\prime}<\right.$ $\left.b \& a^{\prime} \leq a^{\prime} \leq s^{\prime}+u^{\prime} \& t^{\prime}+v^{\prime} \leq b^{\prime}\right\}=\bigvee\left\{\alpha_{0}\left(\left\langle a^{\prime}, b^{\prime}\right\rangle\right) \mid a<a^{\prime}<b^{\prime}<b\right\}$.

Partial proof of 2.2. We shall verify (i) and (viii). It is enough to check that the maps on both sides of these equations agree on generators. For (i), one easily computes $(\alpha \otimes \mathrm{id}) \circ \alpha((a, b))=\bigvee\{(s, t) \otimes(u, v) \otimes(w, x) \mid a \leq s+u+w \& t+v+$ $x \leq b\}=(\operatorname{id} \otimes \alpha) \circ \alpha((a, b))$. As for (viii), after writing out the values of the right and left sides of this equation at a generator $(a, b) \in \mathscr{R}$, one finds that establishing equality depends on showing that certain pairs of sets (e.g., $\{(s, t) \otimes$ $(-\infty, v) \otimes(u, v) \mid a \leq s+u \& t+v \leq b\}$ and $\left\{\left(\max \left\{s_{1}, s_{2}\right\}, \min \left\{t_{1}, t_{2}\right\}\right) \otimes\right.$ $\left.\left.\left(u_{1}, v_{1}\right) \otimes\left(u_{2}, v_{2}\right) \mid t_{1}+v_{1} \leq b \& a \leq s_{2}+u_{2} \& t_{2}+v_{2} \leq b\right\}\right)$ have the same supremum in $\mathscr{R} \otimes \mathscr{R} \otimes \mathscr{R}$. This is not hard.

\section{THE Yosida LOCALE OF AN ABELIAN $l$-GROUP}

We begin by recalling some terms and facts about uniform convergence in an $l$-group. Let $A$ be an abelian $l$-group and let $\left\{a_{i} \mid i=1,2, \ldots\right\}$ be a sequence of elements of $A$. Then $\left\{a_{i}\right\}$ is called s-uniform Cauchy $\left(s \in A^{+}\right)$if for all $m \in \mathbb{N}$ there is $N \in \mathbb{N}$ such that $m\left|a_{n}-a_{n^{\prime}}\right| \leq s$ whenever $n, n^{\prime}>N$. An element $a \in A$ is the s-uniform limit of $\left\{a_{i}\right\}$ if for all $m \in \mathbb{N}$ there is $N \in \mathbb{N}$ such that $m\left|a_{n}-a\right| \leq s$ whenever $n \geq N$, and if $\left\{a_{i}\right\}$ has an $s$-uniform limit, it is said to be s-uniformly convergent. If $\left\{a_{i}\right\}$ is $s$-uniform Cauchy (s-uniformly convergent) for some $s \in A^{+}$, then $\left\{a_{i}\right\}$ is said to be relatively uniform Cauchy 
(relatively uniformly convergent). It is clear that l-homomorphisms preserve relatively uniform Cauchy sequences and relative uniform limits.

We now define the Yosida locale. If $A$ is an abelian $l$-group, let $j: A^{+} \rightarrow$ $\mathscr{Y}(A)$ be the frame freely generated by the set $A^{+}$subject to relations $\left(\mathrm{I}_{1}\right)-\left(\mathrm{I}_{4}\right)$ of Example 1.3 together with the following:

(Y) If $\left\{a_{i} \mid i=1,2, \ldots\right\}$ is increasing and converges relatively uniformly to $a$, then $j(a)=\bigvee\left\{j\left(a_{i}\right) \mid i=1,2, \ldots\right\}$.

If $\varphi: A \rightarrow B$ is an $l$-homomorphism, the map $j_{B} \circ \varphi: A^{+} \rightarrow \mathscr{Y}(B)$ satisfies conditions $\left(\mathrm{I}_{1}\right)-\left(\mathrm{I}_{4}\right)$ and $Y$. Thus, there is a frame morphism $\mathscr{Y}(\varphi): \mathscr{Y}(A) \rightarrow$ $\mathscr{Y}(B)$ with the property that $\mathscr{Y}(\varphi) \circ j_{A}=j_{B} \circ \varphi$. From this follows:

3.1. Proposition. $\mathscr{Y}$ is a (covariant) functor from the category of abelian $l$ groups to Frm.

Note that $\mathscr{Y}(A)$ does not satisfy the relation

$$
\mathrm{\top}=\bigvee\left\{j(a) \mid a \in A^{+}\right\}
$$

nor do we wish to demand this because to do so would prevent $\mathscr{Y}$ from being a functor from $l$-groups to Frm, cf. Example 1.3. In $\mathscr{Y}(A), \bigvee\left\{j(a) \mid a \in A^{+}\right\}$ is strictly less than $T$ and greater than every other element.

\section{Pointed $l$-GROUPS AND THE CONSTRUCTIVE YosidA REPRESENTATION}

The category of abelian l-groups with distinguished positive element and distinguished-element-preserving $l$-homomorphisms is denoted $*-l \mathrm{Ab}$. If $(A, e)$ is an object of $*-l \mathrm{Ab}$, we define

$$
\mathscr{Y}^{*}(A, e)=\mathscr{Y}(A) /(j(e) \sim \mathrm{T}),
$$

i.e., $\mathscr{Y}^{*}(A, e)$ is the frame freely generated by the set $A^{+}$subject to relations $\left(\mathrm{I}_{1}\right)-\left(\mathrm{I}_{4}\right),(\mathrm{Y})$ and the additional relation $j(e)=\mathrm{T}$. From 3.1 we get

4.1. Proposition. $\mathscr{Y}^{*}$ is a (covariant) functor from $*$-lAb to Frm.

In describing the Yosida representation of $(A, e)$, we shall make the assumption that $A$ is divisible. There is actually no loss of generality in doing this because every abelian $l$-group has a divisible hull, see [BKW]. This assumption will greatly simplify the expressions we will be writing. We could avoid the assumption at the expense of making some of the notation more involved and less intuitive, but this would take us well beyond the point of diminishing returns.

We now introduce the functor $C$ from Frm to $*-l \mathrm{Ab}$. We take $C(F)$ to be the l-group $\operatorname{Hom}_{\mathrm{Frm}}(\mathscr{R}, F)$ with distinguished element the unique morphism which has the point $1: \mathscr{R} \rightarrow\{$ as initial factor. Note that $1: \mathscr{R} \rightarrow \notin$ takes $(a, b)$ to the truth value of " $a<1 \& b>1$." In locale language, we are choosing for distinguished element the "constant function 1 on the locale corresponding to $F$."

The Yosida representation is a $*$-lAb morphism $\Phi:(A, e) \rightarrow C\left(\mathscr{Y}^{*}(A, e)\right)$. In order to define it, begin by associating with each $a \in A$ a function $\Phi_{0}(a)$ : $\mathbb{Q} \times \mathbb{Q} \rightarrow \mathscr{Y}^{*}(A, e)$ by putting

$$
\Phi_{0}(a)(\langle p, q\rangle)=j((a-p e) \vee 0) \wedge j((q e-a) \vee 0) \text {. }
$$

4.2. Lemma. For each $a \in A, \Phi_{0}(a)$ satisfies relations $\left(\mathbf{R}_{1}\right)-\left(\mathbf{R}_{5}\right)$ of Example 1.2. Thus $\Phi_{0}(a)$ lifts to a frame morphism $\Phi(a): \mathscr{R} \rightarrow \mathscr{Y}^{*}(A, e)$. 
Proof. Proving $\left(\mathbf{R}_{1}\right)$ and $\left(\mathbf{R}_{2}\right)$ for $\Phi_{0}(a)$ is quite easy, so we omit the details. To show that $\Phi_{0}(a)$ satisfies $\left(\mathbf{R}_{3}\right)$, pick rationals $p, q, r$ and $s$ with $p \leq q<r \leq s$. Let $P=j((a-p e) \vee 0), Q=j((a-q e) \vee 0), R=$ $j((r e-a) \vee 0)$ and $S=j((s e-a) \vee 0)$. Then $P \geq Q$ and $S \geq R$ and it follows that $\Phi_{0}(a)(\langle p, r\rangle) \vee \Phi_{0}(a)(\langle q, s\rangle)=(P \wedge R) \vee(Q \wedge P)=P \wedge S \wedge(R \vee Q)$. Now

$$
\begin{aligned}
R \vee Q & =j((r e-a) \vee(a-q e) \vee 0) \\
& =j\left(\left(\left(\left(\frac{1}{2}(r+q) e-a\right) \vee\left(a-\frac{1}{2}(r+q) e\right)\right)+\frac{1}{2}(r-q) e\right) \vee 0\right) \\
& \geq j\left(\frac{1}{2}(r-q) e\right)=\top,
\end{aligned}
$$

which proves $\left(\mathbf{R}_{3}\right)$. As for $\left(\mathbf{R}_{4}\right)$, one first verifies that $\bigvee\left\{\Phi_{0}(a)\left(\left\langle p^{\prime}, q^{\prime}\right\rangle\right) \mid p<\right.$ $\left.p^{\prime}<q^{\prime}<q\right\}=\bigvee\left\{j\left(\left(a-p^{\prime} e\right) \vee 0\right) \mid p<p^{\prime}\right\} \wedge \bigvee\left\{j\left(\left(q^{\prime} e-a\right) \vee 0\right) \mid q^{\prime}<q\right\}$. Then it remains to show that the right-hand side is equal to $j((a-p e) \vee 0) \wedge j((q e-a) \vee 0)$. We shall show that $\bigvee\left\{j\left(\left(q^{\prime} e-a\right) \vee 0\right) \mid q^{\prime}<q\right\}=j((q e-a) \vee 0)$, leaving the other part for the reader. If $q>q^{\prime}$, then $(q e \vee a)+q^{\prime} e<\left(q^{\prime} e \vee a\right)+q e$, so $(q e \vee a)-\left(a^{\prime} e \vee a\right) \leq\left(q-q^{\prime}\right) e$. This shows that the increasing sequence $\{((q-$ $\left.\left.\left.\frac{1}{n}\right) e-a\right) \vee 0 \mid n=1,2, \ldots\right\}$ converges $e$-uniformly to $(q e-a) \vee 0$, and the desired conclusion follows from relation $(\mathbf{Y})$. Finally, we verify $\left(\mathbf{R}_{5}\right)$. It suffices to show $\bigvee\left\{\Phi_{0}(a)(\langle-n, n\rangle) \mid n=1,2, \ldots\right\}=\mathrm{T}$. Now $\Phi_{0}(a)(\langle-n, n\rangle)=j((a+$ $n e) \vee 0) \wedge j((n e-a) \vee 0)=j(((n e+a) \wedge(n e-a)) \vee 0)=j((n e-|a|) \vee 0)=$ $j\left(\left(e-\frac{1}{n}|a|\right) \vee 0\right)$. Clearly, $\left\{\left(e-\frac{1}{n}|a|\right) \vee 0 \mid n=1,2, \ldots\right\}$ converges $|a|$-uniformly to $e$ so, by $(\mathrm{Y}), \bigvee\left\{\Phi_{0}(a)(\langle-n, n\rangle) \mid n=1,2, \ldots\right\}=j(e)=\mathrm{T}$.

In order to establish the desired properties of the resulting map $\Phi:(A, e) \rightarrow$ $C\left(\mathscr{Y}^{*}(A, e)\right)$, a number of further lemmas are needed.

4.3. Lemma. If $A$ is a divisible abelian l-group, $x, e \in A^{+}$and $n \in \mathbf{N}$, then

$$
((x-n e) \vee 0) \wedge e \leq \frac{1}{n} x \text {. }
$$

Proof. Put $B=x-n e$. By [BKW, 1.3.10 and 1.2.24], $n B \wedge(-B) \leq 0$. Thus $(n B-x) \wedge(n e-x) \leq 0$, so $n(B \wedge e) \leq x$. Thus $n((B \wedge e) \vee 0) \leq x$, and the lemma follows.

4.4. Lemma. If $A$ is an abelian l-group, $z, w \in A, w \geq 0$ and $m \in \mathbb{N}$, then

$$
\bigwedge_{i=-m}^{m}|z-i w| \leq w \vee(|z|-m w)
$$

Proof. The proof is by induction. The case $m=0$ is clear. Let $y \in A$. From the inequality $(y-w) \wedge(w-y) \leq 0$ we get $y \wedge(w-y) \leq w$. From this, it follows that

$$
(w \vee y) \wedge|y-w| \leq w \vee(y-w)
$$

as can be verified by expanding $|y-w|$ and using some distributive laws. By a routine calculation

$$
|z-m w-w| \wedge|z+m w+w|=|| z|-m w-w| .
$$

Thus, setting $y=|z|-m w$ in $(*)$, we have

$$
(w \vee(|z|-m w)) \wedge|z-m w-w| \wedge|z+m w+w| \leq w \vee(|z|-m w-w) .
$$


By the inductive hypothesis, the left side of this inequality majorizes $\bigwedge\{|z-i w| \mid i=-m-1,-m, \ldots, m+1\}$, so we are done.

4.5. Lemma. Suppose $A$ is a divisible abelian l-group, $q \in \mathbb{Q}, n \in \mathbb{N}$, and $a, b \in A$. Let $g=\frac{1}{2}(q e-(a+b)), g_{n}=\bigvee\left\{\left(\left(\frac{1}{2} q+\frac{1}{n}\right) e-a\right) \wedge\left(\left(\frac{1}{2} q-\frac{1}{n}\right) e-b\right) \mid i=\right.$ $\left.-n^{2},-n^{2}+1, \ldots, n^{2}\right\}, H=(g \vee 0) \wedge e$ and $h_{n}=\left(g_{n} \vee 0\right) \wedge e$. Then $0 \leq H-h_{n} \leq \frac{1}{n}\left(e \vee \frac{1}{2}|a-b|\right)$. In other words, $H$ is a relative uniform limit of the increasing sequence $\left\{H_{n}\right\}$, where $H_{n}=\bigvee\left\{h_{j} \mid j=1,2, \ldots, n\right\}$.

Proof. Let $s_{n}=\bigwedge\left\{\left|\frac{1}{2}(a-b)-\frac{1}{n} e\right| \mid i=-n^{2},-n^{2}+1, \ldots, n^{2}\right\}$. By a routine calculation, $g-g_{n}=s_{n}$. In particular, $g-g_{n} \geq 0$, so $0 \leq H-h_{n}$. Also, a direct calculation shows $H-h_{n} \leq\left(s_{n} \vee 0\right) \wedge e$. By 4.4 with $z=\frac{1}{2}(a-b)$, $w=\frac{1}{n} e$, and $m=n^{2}$, we get $s_{n} \leq \frac{1}{n} e \vee\left(\frac{1}{2}|a-b|-n e\right)$. Thus $\left(s_{n} \vee 0\right) \wedge e \leq$ $\frac{1}{n} e \vee\left(\left(\left(\frac{1}{2}|a-b|-n e\right) \vee 0\right) \wedge e\right)$. Applying 4.4 with $x=\frac{1}{2}|a-b|$, we get $\left(s_{n} \vee 0\right) \wedge e \leq \frac{1}{n}\left(e \vee \frac{1}{2}|a-b|\right)$, and we are done.

We now come to the main results of this section.

\subsection{Proposition. If $a, b \in A$, then}

$$
\Phi(a+b)=\Phi(a)+\Phi(b), \quad \Phi(a \vee b)=\Phi(a) \vee \Phi(b), \quad \Phi(e)=1
$$

where the expressions on the right refer to the l-group operations in

$$
\operatorname{Hom}_{\mathrm{Frm}}\left(\mathscr{R}, \mathscr{Y}^{*}(A, e)\right)
$$

induced by the co-l-group operations on $\mathscr{R}$.

Proof. Since elements of the form $(-\infty, q)$ and $(p, \infty)$ generate $\mathscr{R}$, it suffices to check that $\Phi(a+b)$ and $\Phi(a)+\Phi(b) \quad(=\nabla \circ(\Phi(a) \otimes \Phi(b)) \circ \alpha)$ agree at such elements, and that $\Phi(a \vee b)$ and $\Phi(a) \vee \Phi(b)$ do so too. Now $\boldsymbol{\Phi}(a+b)((-\infty, q))=j(((q e-(a+b)) \vee 0)$ and $(\boldsymbol{\Phi}(a)+\boldsymbol{\Phi}(b))((-\infty, q))=$ $\bigvee\{j((t e-a) \vee 0) \wedge j((v e-b) \vee 0) \mid t+v \leq q\}$. Equality in this case is immediate from Lemma 4.5 and the defining relations for $\mathscr{Y}^{*}(A, e)$. The proof of the second assertion is similar but easier and is left to the reader, as is the trivial verification of the third assertion.

We have not, up to now, made explicit mention of any special properties of $\mathscr{Y}^{*}(A, e)$. In fact, this locale is completely regular. To prove this we need to use the well inside relation $a \overline{<} b$ and the really inside relation $a \overline{<} b$, which are defined on pp. 60 and 126 of [J].

\subsection{Proposition. $\mathscr{Y}^{*}(A, e)$ is completely regular.}

Proof. In any frame, $a_{i}<b_{i}, i=1,2$, implies $a_{1} \wedge a_{2}<b_{1} \wedge b_{2}$ and the same is true for the really inside relation. It follows that if each $b_{i}$, $i=1, \ldots, n$, is a supremum of elements really inside itself, then so is $\bigwedge\left\{b_{i} \mid i=1, \ldots, n\right\}$. Obviously, any supremum of elements each of which is expressible as a supremum of elements really inside it is itself expressible in this way. Hence, it suffices to show that any generator $j(a), a \geq 0$, of $\mathscr{Y}^{*}(A, e)$ is a supremum of elements really inside $j(a)$. In fact by relation $Y, j(a)=\bigvee\left\{j\left(\left(a-\frac{1}{n} e\right) \vee 0\right) \mid n=1,2, \ldots,\right\}$, since $\left|\left(\left(a-\frac{1}{n} e\right) \vee 0\right)-a\right| \leq \frac{1}{n} e$. But $j\left(\left(a-\frac{1}{n} e\right) \vee 0\right) \stackrel{\overline{<}}{<}(a)$, as follows by observing that if $p, s, q \in \mathbb{Q}$ and $p>s>q$, then

$$
j((a-q e) \vee 0) \vee j((s e-a) \vee 0)=\top
$$


and

$$
j((a-p e) \vee 0) \wedge j((s e-a) \vee 0)=\perp
$$

so

$$
j((a-p e) \vee 0) \overline{<} j((a-q e) \vee 0) .
$$

Recall that an element $e \in A^{+}$is called a weak unit if $e \wedge a=0$ for all $a \in A^{+}$implies $a=0$. Also, recall that $A$ is said to be archimedean if for all $a, s \in A^{+}, n a \leq s$ for $n=1,2, \ldots$ implies $a=0$.

4.8. Proposition. If $A$ is archimedean and $e \in A^{+}$is a weak unit, then $\operatorname{ker} \Phi=$ $\{0\}$.

Proof. Without necessarily assuming $A$ archimedean, suppose $a \in A^{+}$and $a \in \operatorname{ker} \Phi$. Then for all integers $n>0$,

$$
j\left(\left(a-\frac{1}{n} e\right) \vee 0\right)=\Phi(a)\left(\left(\frac{1}{n},+\infty\right)\right)=\perp .
$$

Since $\left(a-\frac{1}{n} e\right) \vee 0$ converges $e$-uniformly to $a$, we have by $(\mathrm{Y})$ that $j(a)=\perp$. Now suppose $A$ is archimedean with weak unit $e \in A^{+}$. We shall show that $\left\{a \in A^{+} \mid j(a)=\perp\right\}=\left\{0_{A}\right\}$. For this purpose, the site language of [J, pp. 57ff.] is most convenient. View $A^{+}$as a meet semilattice with the infimum operation that of $A$. Relations $\left(\mathrm{I}_{3}\right),\left(\mathrm{I}_{4}\right),(\mathrm{Y})$ and $j(e)=\mathrm{T}$ define a coverage of $A^{+}$in the obvious way and $\mathscr{Y}^{*}(A, e)$ is the frame of $C$-ideals. We must show that $\left\{0_{A}\right\}$ is a $C$-ideal. Now $\left\{0_{A}\right\}$ is clearly closed under sups corresponding to $\left(\mathrm{I}_{3}\right)$ and $\left(\mathrm{I}_{4}\right)$. As for $Y$, if a sequence of $0_{A}$ 's converges relatively uniformly to $a \in A^{+}$, then $m a \leq s \in A^{+}$for all $m=1,2, \ldots$, so $a=0_{A}$. Finally, the relation $j(e)=\top$ means that each $b \in A^{+}$is covered by $b \wedge e=0_{A}$. But if $e$ is a weak unit, then $b \wedge e=0_{A}$ implies $b=0_{A}$.

\section{5. l-IDEALS CLOSED UNDER RELATIVE UNIFORM LIMITS}

The Yosida locale (qua frame) is, by definition, a quotient of the frame of all $l$-ideals. Not surprisingly, this quotient may be described by means of a closure operator on the frame of $l$-ideals. Recall [LZ, p. 427] that an $l$-ideal $I$ of an abelian $l$-group $A$ is said to be uniformly closed if every relatively uniformly convergent sequence in $I$ has all its relatively uniform limits in $I$. Such ideals are significant because an l-group is archimedean if and only if $\{0\}$ is a uniformly closed $l$-ideal, and hence being uniformly closed is equivalent to being the kernel of an $l$-homomorphism to an archimedean $l$-group. Below, we consider the set of all uniformly closed $l$-ideals of $A$ to be partially ordered by inclusion. Since an intersection of uniformly closed $l$-ideals is uniformly closed, for every element of $A$ there is a smallest uniformly closed $l$-ideal containing that element.

The reader is reminded that as of this section, no attempt is made to produce constructive proofs.

5.1. Proposition. There is an order-preserving bijection between $\mathscr{Y}(A) \backslash\{T\}$ and the set of all uniformly closed l-ideals of $A$ in which $j(a)$ corresponds to the smallest uniformly closed l-ideal containing $a$.

Proof. If $J$ is any $l$-ideal of $A$, let $J^{*}$ denote the set of all $x \in A$ such that $|x|$ is less than the relatively uniform limit of some relatively uniformly convergent increasing sequence of elements of $J$. Note that $J^{*}$ is an $l$-ideal. Define an 
ordinal sequence by setting $J_{0}=J, J_{\alpha+1}=\left(J_{\alpha}\right)^{*}$ and $J_{\lambda}=\bigcup_{\alpha<\lambda} J_{\alpha}$ for limit ordinals $\lambda$. The sequence eventually stabilizes. Let $n(J)$ be the stable value. Since $n(J)=(n(J))^{*}, n(J)$ is the smallest uniformly closed $l$-ideal containing $J$, by [LZ, 60.2]. Using the easily verified fact that $\left(J_{1} \cap J_{2}\right)^{*}=J_{1}^{*} \cap J_{2}^{*}$, it is seen that $n\left(J_{1} \cap J_{2}\right)=n\left(J_{1}\right) \cap n\left(J_{2}\right)$; furthermore $J \subseteq n(J)=n(n(J))$. Thus $n$ is a nucleus on $l-\operatorname{Idl}(A)$. It is easy to see that the congruence on $l-\operatorname{Idl}(A)$ corresponding to $n$ is precisely that generated by the relation $Y$.

5.2. Corollary. If $A^{\prime}$ is the archimedean reflection of $A$ (i.e., $A^{\prime}=A / I$, where $I$ is the smallest uniformly closed l-ideal of $A)$ then $\mathscr{Y}(A) \cong \mathscr{Y}\left(A^{\prime}\right)$.

5.3. Corollary. There is an order-preserving bijection between $\mathscr{Y}^{*}(A, e)$ and the set of all uniformly closed l-ideals of $A$ contained in the uniformly closed l-ideal generated by $e$.

The following example shows that even when $e$ is a weak unit, there may be a proper uniformly closed $l$-ideal containing $e$. Let $A$ be the $l$-group of all (finitely) piecewise linear functions on $[0,1]$. Then the function $f(x)=x$ is a weak unit, but the $l$-ideal it generates is the kernel of the map $A \rightarrow \mathbb{R}$ which sends a function to its value at 0 . On the other hand, if $A$ is an archimedean $f$-ring with unity $e$, then $e$ is contained in no proper uniformly closed $l$-ideal. To see this, let $n \in \mathbb{N}, a \in A^{+}$. From $a^{2}-2 n a+n^{2} e=(a-n e)^{2} \geq 0$, it follows that $\frac{1}{n} a^{2} \geq(2 a-n e) \vee 0=|2 a-(2 a \wedge n e)|$. Hence $2 a$ is the $a^{2}$-uniform limit of the sequence $2 a \wedge n e$ which is in the convex $l$-subgroup generated by $e$.

\section{Relationships WITH THE Classical Yosida SPACE}

In this section, we find generators and relations for the topology of the classical Yosida space $X(A, e)$ of a pointed $l$-group $(A, e)$. This is then used to show that $\mathscr{Y}^{*}(A, e)$ is the quotient of the frame of opens of $X(A, e)$ corresponding to the localic intersection of the domains of reality of the functions on $X(A, e)$ representing $A$, demonstrating that $\mathscr{Y}^{*}(A, e)$ is indeed the same as the Yosida locale defined in $\left[\mathrm{MV}_{2}\right]$.

Let $(A, e)$ be an archimedean $l$-group with weak unit $e$. The classical Yosida theorem provides a canonical identification of $(A, e)$ with an $l$-group of extended real-valued functions on the compact space $X(A, e)$ of $e$-maximal $l$-ideals of $(A, e)$. Under this identification the following are satisfied: each $a \in A$ is real-valued on a dense subset of $X(A, e), A$ separates the points of $X(A, e)$ and $e$ corresponds to the constant function 1. This identification is essentially unique, in that any identification of $(A, e)$ with an l-group of extended real-valued functions on a compact space $X^{\prime}$ which satisfies the above conditions is induced from the canonical one by a homeomorphism of $X(A, e)$ with $X^{\prime}$. Below, we shall treat $(A, e)$ as a sub-l-group of $D(X(A, e))=$ the extended real-valued functions on $X(A, e)$.

Let $\mathscr{X}(A, e)$ be the frame freely generated by the symbols $k(a), a \in A^{+}$, subject to the following relations: $\left(\mathrm{I}_{1}\right)-\left(\mathrm{I}_{4}\right)$ of Example 1.3, $k(e)=T$ and the relations:

(X) if $\left\{a_{i} \mid i=1,2, \ldots\right\}$ is increasing and converges $e$-uniformly to $a$, then $k(a)=\bigvee\left\{k\left(a_{i}\right) \mid i=1,2, \ldots\right\}$.

6.1. Proposition. The rule $k(a) \rightarrow \operatorname{coz}(a) \subseteq X(A, e)$ induces an isomorphism of $\mathscr{X}(A, e)$ with the frame of opens of $X(\bar{A}, e)$. 
Proof. Since the map $a \rightarrow \operatorname{coz}(a)\left(a \in A^{+}\right)$satisfies the relations defining $\mathscr{X}(A, e)$ it indeed induces a frame map from $\mathscr{X}(A, e)$ to the opens of $X(A, e)$. This is obviously surjective, since the cozero sets of $A$ are an open base for $X(A, e)$. It remains to show that the map is injective. By the proof of $4, \mathscr{X}(A, e)$ is a regular frame. It is a property of regular frames that every congruence is generated by open congruences, and since an open congruence is generated by the identification of some element with $T$, we just have to check that if $\bigcup_{\alpha} \operatorname{coz}\left(a_{\alpha}\right)=X(A, e)$ then $\bigvee_{\alpha} k\left(a_{\alpha}\right)=\mathrm{T}$. By compactness, $\bigcup_{\alpha} \operatorname{coz}\left(a_{\alpha}\right)=\operatorname{coz}\left(a_{1}\right) \vee \cdots \vee \operatorname{coz}\left(a_{n}\right)$ for some $a_{1}, \ldots, a_{n}$ among the $a_{\alpha}$. Then $e \leq \sum n_{i} a_{i}$ for appropriate constraints $n_{i}$, so $\bigvee k\left(a_{i}\right)=T$, by $\left(\mathrm{I}_{3}\right)$.

The relations defining $\mathscr{Y}^{*}(A, e)$ are obviously stronger than those defining $\mathscr{X}(A, e)$, so $\mathscr{Y}^{*}(A, e)$ is a quotient of $\mathscr{X}(A, e)$. Since $\mathscr{X}(A, e)$ is regular, the kernel of the projection $\mathscr{X}(A, e) \rightarrow \mathscr{Y}^{*}(A, e)$ is determined by the elements of $\mathscr{X}(A, e)$ sent to $\mathrm{T}$. For $s \in A^{+}$, define $\operatorname{fin}(s) \in \mathscr{X}(A, e)$ by $\operatorname{fin}(s)=\bigvee_{n=1}^{\infty} k((n e-s) \vee 0)$. Obviously, fin $(s)$ corresponds to the dense open $\{x \in X(A, e) \mid s(x) \in \mathbb{R}\}$. Let $\operatorname{Fin}(A, e)$ denote the least congruence on $\mathscr{X}(A, e)$ containing $\left\{\operatorname{fin}(s) \sim T \mid s \in A^{+}\right\}$.

6.2. Proposition. The kernel of the projection $\mathscr{X}(A, e) \rightarrow \mathscr{Y}^{*}(A, e) ; k(a) \rightarrow$ $j(a)$ is $\operatorname{Fin}(A, e)$.

Proof. Since the sequence $\left\{\left(e-\frac{1}{n} s\right) \vee 0 \mid n=1,2, \ldots\right\}$ converges $s$-uniformly to $e$, the kernel clearly contains $\operatorname{Fin}(A, e)$. To show that $\operatorname{Fin}(A, e)$ contains the kernel, it suffices to show that $\mathscr{X}(A, e) / \operatorname{Fin}(A, e)$ satisfies the relations $Y$. Suppose that $\left\{a_{i} \mid i=1,2, \ldots\right\} \subseteq A^{+}$is increasing and converges $s$-uniformly to $a$. We want to show $\bigvee k\left(a_{i}\right) \equiv k(a)(\bmod \operatorname{Fin}(A, e))$. For $a, b, x, s \in$ $A^{+}$, whenever $a-b \leq \frac{1}{m} s$ we have

$$
a \wedge((x-s) \vee 0)-b \wedge((x-s) \vee 0) \leq \frac{1}{m} x
$$

as can be checked by verifying in $\mathbb{Z}$ or (with a little more work) writing a direct proof. Setting $x=n e$ and letting $b=a_{i}$ in this inequality shows that $a_{i} \wedge((n e-s) \vee 0)$ converges $e$-uniformly to $a \wedge((n e-s) \vee 0)$. Thus, by using the frame distributive law and the defining identifies for $\mathscr{X}(A, e)$

$$
k((n e-s) \vee 0) \wedge \bigvee k\left(a_{i}\right)=k((n e-s) \vee 0) \wedge k(a)
$$

Using distributivity once again

$$
\operatorname{fin}(s) \vee \bigvee k\left(a_{i}\right)=\operatorname{fin}(s) \wedge k(a)
$$

By 6.2, we may apply any of the facts about the Yosida locale proved in $\left[\mathrm{MV}_{2}\right]$ to $\mathscr{Y}^{*}(A, e)$. In particular, a lemma of Isbell $\left(2.11\right.$ of $\left.\left[\mathrm{MV}_{2}\right]\right)$ says that archimedean $A$ is closed under countable composition if and only if the localic Yosida representation $\Phi: A \rightarrow \operatorname{Hom}\left(\mathscr{R}, \mathscr{Y}^{*}(A, e)\right)$ is surjective. Since $A$ is an $f$-ring when it is closed under countable composition, the remarks at the end of $\S 5$ together with 5.3 show

6.3. Corollary. Archimedean l-groups with weak unit which are closed under countable composition are isomorphic if and only if their frames of uniformly closed l-ideals (archimedean kernels) are isomorphic. 


\section{RELATIONS WITH $\sigma$-FRAMES}

The relevance of $\sigma$-frames to the topics with which this paper is concerned has been made clear in $\left[\mathrm{MV}_{1}\right.$ and $\left.\mathrm{MV}_{2}\right]$. The definition of the Yosida locale by generators and relations provides an interesting perspective. Since the defining relations involve only countable suprema, there is a reasonable sense in which the uncountable suprema in $\mathscr{Y}^{*}(A, e)$ ought to be superfluous. It is possible to use $\sigma$-frames to make this intuition precise. On the other hand, $\sigma$-frames are not easily dealt with constructively because cardinality considerations that arise frequently become extremely tricky without choice. This is the reason that we have used frames rather than $\sigma$-frames in the localic Yosida theorem presented in $\S 4$. Accepting convenient set-theoretic axioms at this point, however, the $\sigma$-frame version of the localic Yosida theorem can be obtained by trivial modification of the frame proof, as shown below.

We shall recall some basic facts. A $\sigma$-frame is a lattice with top and bottom equipped with a countable join operation $\bigvee$ which satisfies the distributive law $a \wedge \bigvee B=\bigvee\{a \wedge b \mid b \in B\}$ for all elements $a$ and countable subsets $B$. The $\sigma$ frame morphisms preserve top, bottom, finite meets and countable joins. The category of $\sigma$-frames is varietal (albeit with an infinitary operation). Consequently, limits in the category of $\sigma$-frames are constructed in the category of sets, all (small) colimits exist, monomorphisms are injective, ... etc., see [ $\mathrm{J}, \mathrm{p}$. 23]. Most important here, the free $\sigma$-frame on any set exists and $\sigma$-frames, like frames, can be presented by generators and relations.

Let $A$ be an abelian $l$-group and $e \in A^{+}$. Define $\sigma-\mathscr{Y}(A)$ to be the $\sigma$ frame with the same generators and relations as $\mathscr{Y}(A)$ and define $\sigma-\mathscr{Y}^{*}(A, e)$, $\sigma-\mathscr{X}(A, e)$ and $\sigma-\mathscr{R}$ analogously. Note that since $\mathscr{R}$ is countably generated, $\sigma-\mathscr{R}$ and $\mathscr{R}$ have the same elements.

It is easy to see that $\sigma-\mathscr{Y}$ is a functor from $l$-groups to $\sigma$-frames and that $\sigma-\mathscr{Y}^{*}$ and $\sigma-\mathscr{X}$ are functors from pointed $l$-groups to $\sigma$-frames. Since the proofs of $2.2,4.2,4.3,4.7,4.8$ and 6.2 involve only countable suprema, the obvious analogues for $\sigma$-frames are true. In particular, $\sigma-\mathscr{X}(A, e)$ and $\sigma-\mathscr{Y}^{*}(A, e)$ are regular. Since a regular $\sigma$-frame is normal, 4.7 can actually be strengthened.

An element $x$ of a frame is called countable if $x \leq \bigvee B$ implies $x \leq B^{\prime}$ for some countable subset $B^{\prime} \leq B$. Noting that $\sigma-\mathscr{Y}^{*}(A, e)$ and $\sigma-\mathscr{X}(A, e)$ may be identified with the subsets of $\mathscr{Y}^{*}(A, e)$ and $\mathscr{X}(A, e)$ accessable as countable suprema of generators, it is trivial to deduce that these are exactly the countable elements of $\mathscr{Y}^{*}(A, e)$ and $\mathscr{X}(A, e)$. In particular, from results in $\left[\mathrm{MV}_{1}\right]$, it follows that $\mathscr{Y}^{*}(A, e)$ is $\sigma$-coherent, hence Lindelöf.

Recall that coz denotes the functor from frames to $\sigma$-frames which associates with a frame its $\sigma$-frame of cozero elements. From 2.1 of $\left[\mathrm{MV}_{1}\right]$, and 3.1 of $\left[\mathrm{MV}_{2}\right]$, we get

7.1. Proposition. (a) The functor $\sigma-\mathscr{X}$ is naturally equivalent to $\operatorname{coz} \circ \mathscr{X}$, so in particular $\sigma-\mathscr{X}(A, e)$ is isomorphic to the $\sigma$-frame of cozero sets of $X(A, e)$.

(b) The functor $\sigma-\mathscr{Y}^{*}$ is naturally equivalent to $\operatorname{coz} \circ \mathscr{Y}^{*}$.

(c) $\sigma-\mathscr{Y}^{*}$ and $\operatorname{Hom}_{\sigma \text {-frame }}(\sigma-\mathscr{R}, \ldots)$ give an equivalence between the category of regular $\sigma$-frames and the category of weak-unital archimedean l-groups closed under countable composition.

Part (c) fulfills a promise made in $\left[\mathrm{MV}_{2}\right]$ just after the statement of Proposition 3.1 . 


\section{REFERENCES}

[B] S. J. Bernau, Free abelian lattice groups, Math. Ann. 180 (1969), 48-59.

[BKW] A. Bigard, K. Keimel, and S. Wolfenstein, Groupes et anneaux réticulés, Lecture Notes in Math., vol. 608, Springer-Verlag, 1977.

[BM] B. Banaschewski and C. Mulvey, Stone-Čech compactification of locales. II, J. Pure Appl. Algebra 33 (1984), 107-122.

[FH] M. P. Fourman and J. M. E. Hyland, Sheaf models for analysis, Applications of Sheaves, Lecture Notes in Math., vol. 753, Springer-Verlag, 1979, pp. 280-301.

[HM] A. W. Hager and J. J. Madden, Majorizing-injectivity in abelian lattice-ordered groups, Rend. Sem. Mat. Univ. Padova 69 (1983), 181-194.

[J] P. T. Johnstone, Stone spaces, Cambridge Studies in Advanced Math. No. 3, Cambridge Univ. Press, 1982.

[JT] A. Joyal and M. Tierney, An extension of the Galois theory of Grothendieck, Mem. Amer. Math. Soc., no. 309, 1984.

[LZ] W. A. J. Luxemburg and A. C. Zaneen, Riesz spaces, I, North-Holland, Amsterdam, 1971.

[M $\left.\mathrm{M}_{1}\right] \quad$ J. J. Madden, On the Yosida representation, General Topology and Applications: Proceedings of the 1988 Northeast Conference, edited by Rae Michael Shortt, Marcel Dekker, 1990, pp. 183-192.

$\left[\mathrm{M}_{2}\right] \quad$ J. J. Madden, $\kappa$-frames, J. Pure Appl. Algebra 70 (1991), 107-127.

$\left[\mathrm{MV}_{1}\right]$ J. J. Madden and J. Vermeer, Lindelöf locales and real compactness, Math. Proc. Cambridge Philos. Soc. 99 (1986), 473-480.

$\left[\mathrm{MV}_{2}\right]$, Epicomplete archimedean l-groups via a localic Yosida theorem, J. Pure Appl. Algebra 68 (1990), 243-252.

[Mu] C. J. Mulvey, A syntactic construction of the spectrum of a commutative $C^{*}$-algebra, Tagungsbereicht of Oberwolfach Category Meeting, 1977.

Department of Mathematics, Indiana University at South Bend, South Bend, Indiana 46634

Current address: Department of Mathematics, Louisiana State University, Baton Rouge, Louis iana 70803-0001 